\title{
A mechanistic and experimental study on the diethyl ether oxidation
}

\author{
S. Di Tommaso ${ }^{1-2}$, P. Rotureau ${ }^{2 *}$, B. Sirjean ${ }^{3}$, R. Fournet ${ }^{3}$, W. Benaissa ${ }^{2}$, P. Gruez $^{2}$, C. \\ Adamo ${ }^{1}$ \\ ${ }^{1}$ Laboratoire d'Electrochimie, Chimie des Interfaces et Modélisation pour l'Energie, CNRS \\ UMR 7575, Chimie ParisTech, 11 rue Pierre et Marie Curie, F-75231 Paris Cedex 05, France \\ ${ }^{2}$ INERIS, Parc Technologique Alata - BP 2 - 60550 Verneuil-en-Halatte, France \\ ${ }^{3}$ Laboratoire Réactions et Génie des Procédés, Université de Lorraine - CNRS, 1, rue Grandville, \\ BP 20451, Nancy, France
}

*Corresponding author: patricia.rotureau@ineris.fr; tel:+33(0)344556329;fax:+33(0)344556565

Keywords: diethyl ether (DEE) - DFT - kinetics model - oxidation at accelerated conditions

\begin{abstract}
This work presents the results of the theoretical investigations on autoxidation process of diethyl ether (DEE), a chemical largely used as solvent in laboratories and considered to be responsible for various accidents. Based on Density Functional Theory (DFT) calculations, the aims of this study were the identification of all the most probable reaction paths involved in DEE oxidation (at ambient temperature and under conditions that reflect normal storage conditions) and the characterization of products and all potential hazardous intermediates, such as peroxides. Results indicate that industrial hazards could be related to hydroperoxide formation and accumulation during the chain propagation step. A detailed kinetics model of DEE oxidation in the gas phase was then developed from all energetic and kinetics parameters collected during the mechanistic study. Outputs of the kinetics model, in terms of time of evolution of product concentrations, have been then compared with the experimentally measured concentration of products (notably hydroperoxides) issued from tests on DEE oxidation conducted under accelerated conditions with autoclaves.
\end{abstract}

\section{Introduction}

A great number of organic compounds spontaneously decompose by a free-radical reaction of the carbon chain with molecular oxygen, in a self-propagating process of auto-oxidation that may generate a large variety of peroxide molecules. Many laboratory accidents can be ascribed to the presence of such peroxides in stored chemicals [1-4], often under poor conditions, for over long periods. Ethers are typical solvents showing such a behavior. Dimethyl ether (DME) is the most investigated ether of this class both experimentally [5,6] and theoretically [7], since it has a relevant role, due to its good ignition properties, in the domain of biofuels. In particular, Andersen and coworkers [7] proposed a DFT study on the process of low temperature oxidation 
of this ether. Experimental studies also exist on the oxidation of the other short-chains ethers suggesting oxidation products or intermediates or giving some hints on the kinetics of the process. It is the case of diethyl ether (DEE) [8-12], diisopropyl ether (DIPE) [11, 13] or methyl tert-butyl ether (MTBE) [14]. However few investigations have been devoted to the mechanistic elucidation of their oxidation under usual storage conditions [6, 16]. Moreover, the chemistry of the oxidation of saturated branched ethers has some similarities with the well known of alkanes [15], as suggested for DME by Andersen and Carter [6]. This has also been demonstrated for diethyl ether in our latest extensive theoretical study on its low temperature and low pressure oxidation [16].

In this scientific context, where incomplete experimental data are available and only a sketchy picture of the process emerges, the present work proposes an in-depth study of DEE oxidation carried out at both theoretical and experimental levels to better understand the process and manage the accidental risks linked to diethyl ether use and storage. In the following, starting from a brief presentation of the results of the mechanistic study (based on quantum chemistry calculations) of all the possible reactions involved in the process, the development and the validation of the kinetics model obtained for the gas phase oxidation of DEE will be discussed. At the end, the issues of kinetics model, in terms of concentrations of products and time of reactions, will be compared with experimental data obtained on DEE oxidation tests carried out in accelerated conditions.

\section{Materials and Methods}

\subsection{Molecular modeling}

All the Density Functional Theory (DFT) calculations have been performed using the B3LYP functional [17] and Gaussian 03 program [18]. The 6-31+G(d,p) basis set was used to optimize structures and for subsequent frequency calculations to characterize stationary points as minima or transition states. Intrinsic reaction coordinate (IRC) calculations were also performed to verify that identified products and reactants were correctly connected [19]. For radicals (open shell systems), unrestricted DFT calculations were carried out and the obtained results were checked for wave-function instability.

Enthalpy values relative to reactions discussed in the following are collected as activation barriers $\left(\Delta \mathrm{H}^{\ddagger}\right)$ and product stabilization $(\Delta \mathrm{H})$ in table I. The relative enthalpies are referred to a single reaction step, that is reactants-products or reactants-transition state.

\subsection{Rate constants calculations}

Rate constants for each elementary reaction were calculated using classical transition state theory (TST) [20]. The tunneling effect was taken into account for reactions involving inter or intramolecular hydrogen transfer using the transmission coefficient in the form proposed by Wigner [21] or Skodje and Truhlar [22] or Eckart [23]. Activation enthalpies have been computed as the difference between enthalpies of formation (with respect to isolated atoms) of all reactants and related transition states. The kinetic parameters were obtained by fitting the rate constant values obtained from TST at several temperatures between 500 and $2000 \mathrm{~K}$ with the equation

$$
k_{\infty}=
$$

Where $A, n$, and $E a$ are the parameters of the modified Arrhenius equation and $k_{\infty}$ is the highpressure-limit rate constant. 


\subsection{Experimental tools}

Storage of chemicals is generally performed at room temperature. Under these conditions, the kinetics are slow and real time tests can take several weeks. As the kinetics are accelerated by temperature, an experimental setting has been designed in order to store DEE at high temperature. Contrary to Naito et al. [11] who used Accelerated Rate Calorimeter (ARC) bombs of $10 \mathrm{~mL}$, experiments were carried out using six $50 \mathrm{~mL}$ autoclaves: each stainless steel vessel had previously undergone a passivating step and equipped with a temperature sensor and a pressure transducer. The six autoclaves are inserted in a heating block controlling the temperature (cf. figure 1).

Diethyl ether (99.7\%) was obtained from VWR and was used without further addition of a stabilizer. Fourteen milliliters of DEE were introduced into each autoclave and pressurized by synthesized air from a gas cylinder at an approximately pressure of 10 bar absolute. The samples were then heated to two temperatures: $80^{\circ} \mathrm{C}$ and $100^{\circ} \mathrm{C}$. Temperatures and pressures inside the autoclaves were recorded throughout the experiment.

At determined storage times, the samples were recovered in order to determine the amount of peroxide formed. Concentration of peroxide was measured using a potentiometric titrator and a solution of potassium permanganate. This method gives the global amount of peroxide function (equivalent $\mathrm{H}_{2} \mathrm{O}_{2}$ ).

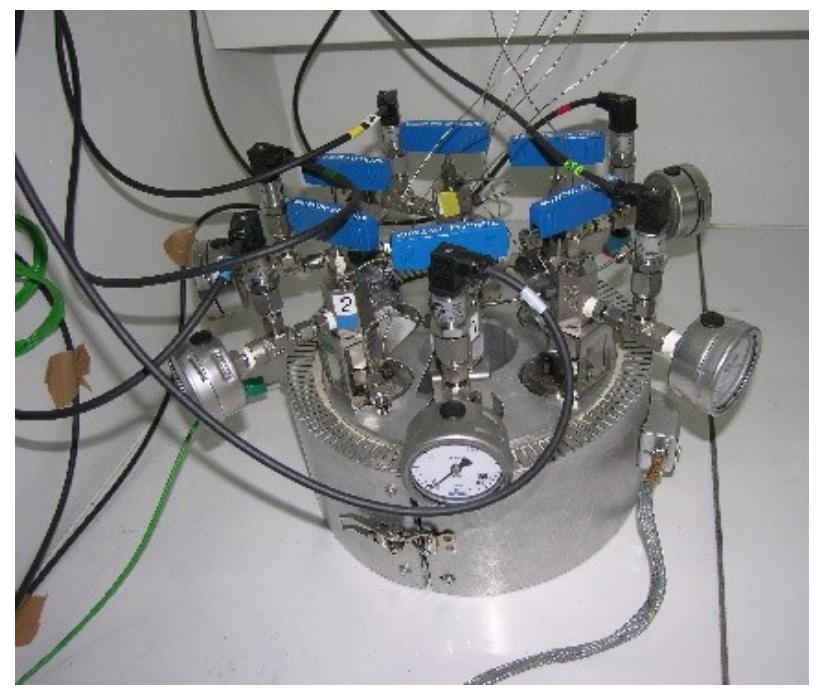

Figure 1.Experimental setting for experiments at accelerated conditions

\section{Results and discussions}

\subsection{Molecular modeling}

In figure 2 is depicted a scheme summarizing the main reaction pathways of the diethyl ether (R) oxidation issued of DFT mechanistic study of the process [16]. Analogously to the accepted alkane autoignition mechanism $[15,24]$ the oxidation process starts with an initiation step in which a radical $\mathbf{R} \cdot$ is produced. This species can simply undergo decomposition. Alternatively, a chain propagating cycle is established if the $\mathbf{R} \cdot$ radical reacts with molecular oxygen to give a ROO- species. This peroxy radical can then react with a new DEE molecule to produce a hydroperoxide $\mathbf{R O O H}$ and regenerate a R. radical. Chain termination ensues when two radical intermediates couple to each other (e.g. dimerization) with loss of the radical character. By 
contrast, radical isomerization may occur without interrupting the chain reaction. This schematic picture is complicated by the possible decomposition of the hydroperoxides that represent the stoichiometric products of the ideal chain-propagating cycle.

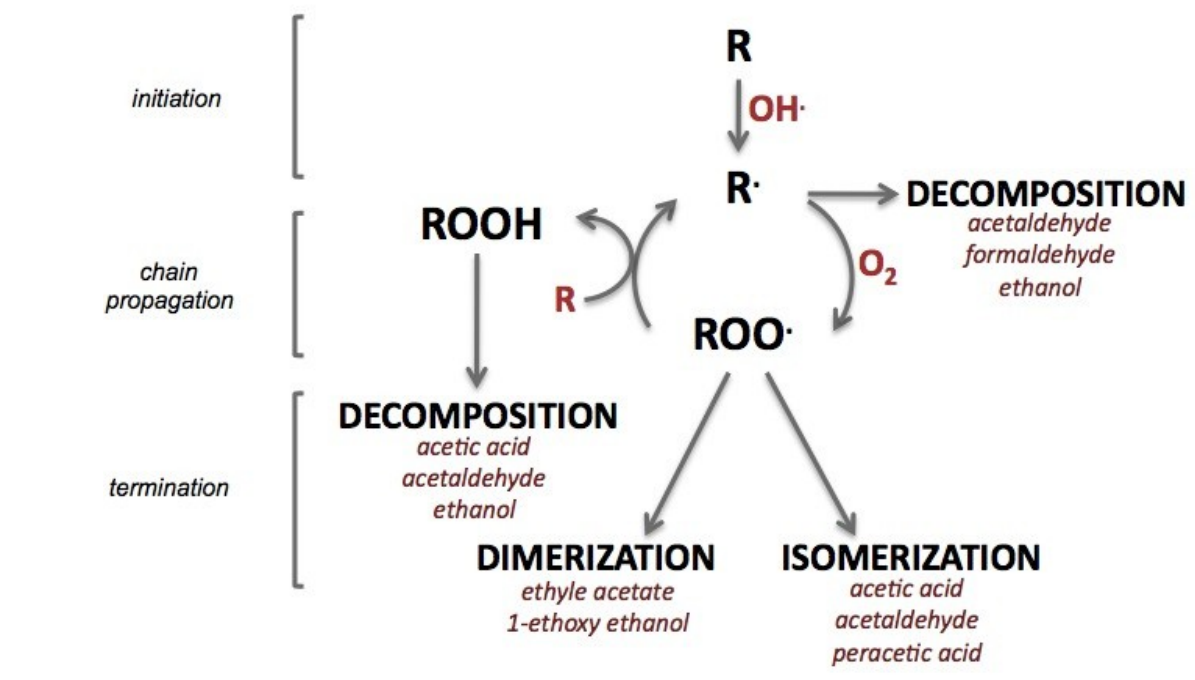

Figure 2.Global scheme of the proposed mechanism of DEE (R) oxidation.

The experimentally observed products are also reported

Among the various chain initiators that have been proposed in literature, $\mathrm{OH}$ radical is, from our calculations, the most efficient one from an energy point of view [16]. Competition among different pathways starts with the formation of the radical $\mathbf{R} \cdot$. In conditions of low (null) oxygen concentration, its decomposition, characterized by a high rate constant $\left(\mathrm{k}=1.29 \mathrm{~s}^{-1}\right.$ at $298 \mathrm{~K}$, as reported in figure 3), leads to the formation of acetaldehyde, formaldehyde and ethanol. In presence of oxygen, the production of peroxy radicals (ROO-) becomes important and the corresponding reaction is strongly exothermic $(\Delta \mathrm{H}=-31.8 \mathrm{kcal} / \mathrm{mol})$ and barrierless. The resulting ROO - radicals can evolve along three competitive reaction pathways: hydroperoxide production, isomerization and dimerization (see figure 2).

Hydroperoxide production is endothermic by $12.8 \mathrm{kcal} / \mathrm{mol}$ and has an activation barrier of approximately $13.3 \mathrm{kcal} / \mathrm{mol}$. Nonetheless, it becomes probable in the usual conditions of autoxidation, where DEE concentration is high. Indeed, this reaction channel leads to some of the major products identified experimentally (acetic acid, acetaldehyde, ethanol) [10]. On the other hand, the isomerization reaction features significant product stabilization and activation energies comparable to that of hydroperoxide production. Moreover, it could account for the formation of some of the observed products (acetaldehyde, acetic acid, peracetic acid) [10]. However the two pathways are characterized by different entropic effects, so that the final Gibbs free energies are 23.7 and $19.3 \mathrm{kcal} / \mathrm{mol}$ for the hydroperoxide production and isomerization, respectively. These different values of Gibbs free energy enthalpy lead to a very important difference in rate constant values, as reported in figure 3.

Finally, dimerization is thermodynamically favored due to significant product stabilization. However, the activation energies along the following steps of these paths are higher than in other reactions, the lowest energy barrier being $32.3 \mathrm{kcal} / \mathrm{mol}$. Furthermore, dimerizations are bimolecular reactions, and their kinetics are therefore highly sensitive to the concentration of peroxy radicals in the reaction medium. In fact the dimerization pathway has been suggested in 
literature as the major reaction channel for DEE oxidation in atmospheric conditions [12]. In solution, dimerization happens if the two peroxy radicals are close, so that the reaction is limited by the diffusion of two highly reactive species: this channel could be considered as improbable.

Therefore, among all the characterized pathways, decomposition of $\mathbf{R} \cdot$ and isomerization could be the most relevant in solution.

Moreover, from a hazard point of view, different hydroperoxides have been identified as products or sub products of principal reaction pathways. Considering the high activation barriers for decomposition of these compounds, their accumulation during DEE oxidation could be a (major) risk source during the storage of the chemical.

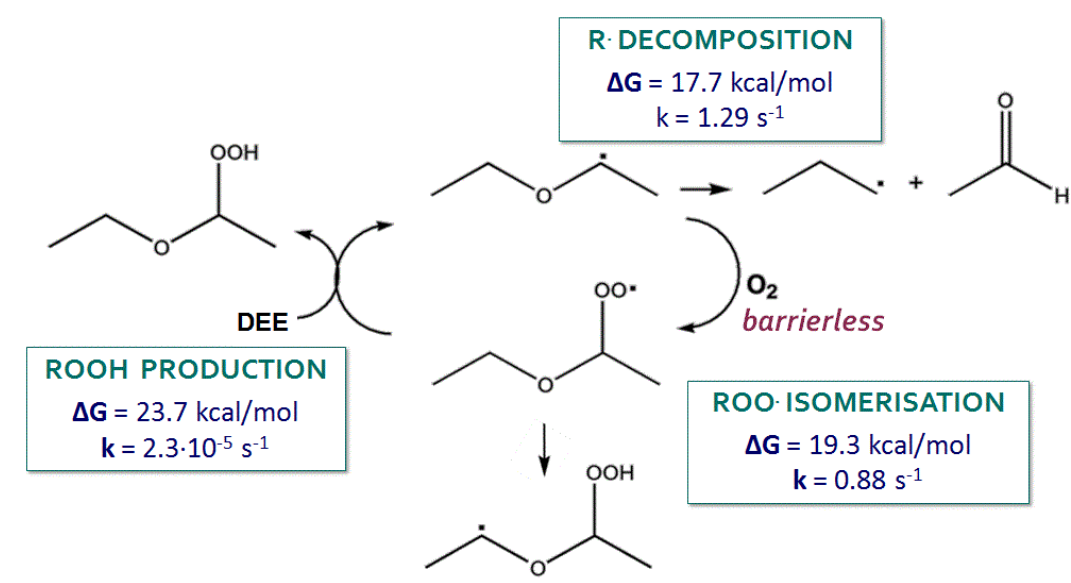

Figure 3. Sketch of the principal reaction pathways identified.

For each reaction the activation free Gibbs energy and the rate constant are specified

\subsection{Detailed chemical kinetics model}

The approach used for the development of the chemical kinetics model of the process is summarized in figure 4 .

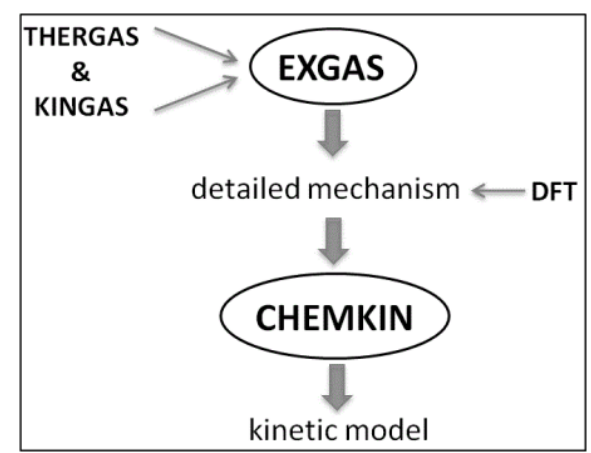

Figure 4. Scheme of the approach used for the development of the kinetics model

The first step was the automatic generation of a detailed mechanism of the oxidation process of DEE with the software EXGAS [25]. The mechanism produced contains thermochemistry of each species involved in the process in the form of coefficients of NASA polynomial (calculated by the software THERGAS [26] based on Benson group additivity methods) and the kinetics of 
each reaction considered (calculated by the software KINGAS). All thermodynamic and kinetics data issued from the DFT mechanistic study [16] were introduced in this mechanism. The final DEE oxidation model contains 158 species and 355 reactions. Simulations were performed using the SENKIN code of the CHEMKIN II [27] program suite assuming homogeneous gas-phase reactions in an isochoric static reactor. Temperature and pressure conditions can be selected as inputs to match target experimental data.

The mechanism produced was developed and validated by the comparison with experimental literature data in terms of evolution of pressure and product concentration. In particular, because of the lack of experimental data on the DEE oxidation in the liquid phase at room temperature, the model was developed for the process in the gas phase using experimental data (concentrations of products in function of time at $153^{\circ} \mathrm{C}$ ) from experimental work of Waddington [10]. It was then validated from comparison with the Lemay and Ouellet study [9] performed at a different temperature than Waddington. Figure 5 reports the comparison between experimental data obtained by Lemay and Ouellet [9] in their study on DEE gas phase oxidation at $168^{\circ} \mathrm{C}$ and the curves generated from the CHEMKIN simulation (at $\mathrm{T}=172^{\circ} \mathrm{C}$ ) in the developed kinetics model.

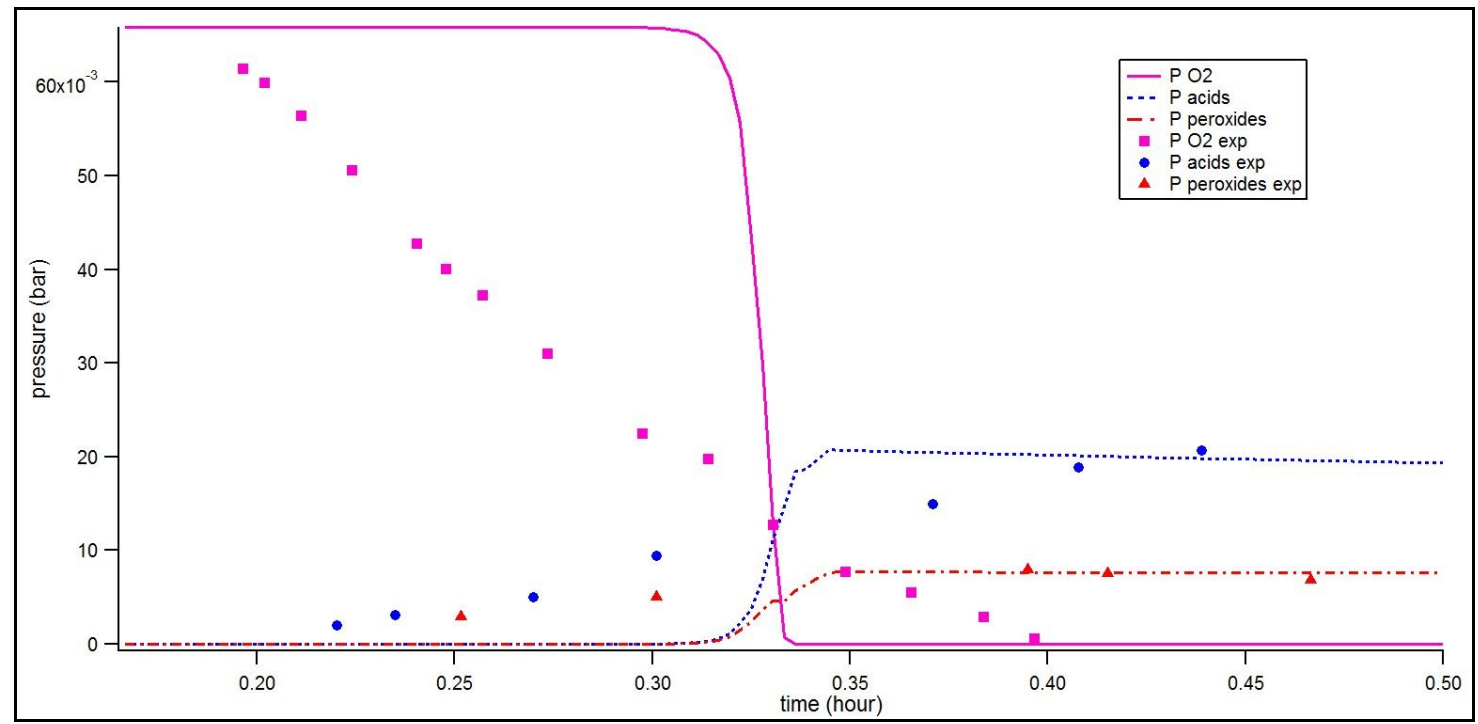

Figure 5. Evolution of partial pressures of oxygen, acids and peroxides at $T=172^{\circ} \mathrm{C}$ by CHEMKIN simulation. Experimental curves [9] have been displaced by about 10 minutes to facilitate the comparison

Some considerations can be made on the basis of this graph:

- A displacement in the observation of reactivity of the mixture between experiment and simulations is observed. In particular DEE is consumed earlier (approximately 10 minutes) in the experiment at a lower temperature $\left(168^{\circ} \mathrm{C}\right.$ instead of $172^{\circ} \mathrm{C}$ in simulation). We have to note that experimental parameters are not accurately detailed in the studies $[9,10]$ used as references for the development of the kinetics model. If we consider temperature, for instance, in both cases given values are referred to the temperature of the oven in which the reactor is suspended and not to the one in the reactor, and there is no reference to the adiabaticity of the reaction vessel. 
- After the beginning of reactivity, the trends are well reproduced by the kinetics model as well as the selectivity of the mixture of products.

- In the first period of the process, the induction time, is probably characterized by heterogeneous reactions with the reactor surface, which are not taken into account in simulations.

Therefore we can conclude that the model is validated for DEE oxidation in the gas phase in terms of reproduction of product concentrations. Because of ill-defined experimental conditions in the reactor and interferences of heterogeneous wall reactions, it is difficult to make firm conclusions about the difference of reactivity observed between experiments and simulations. Consequently, temperature and residence time adjustments remain acceptable.

\subsection{Experimental results}

The peroxide concentration vs. time profiles are given in figure 6 . These results show two tendencies: initially the peroxide concentration increases to a maximum and then decreases to reach very low values. At $80^{\circ} \mathrm{C}$, the maximum is around $6000 \mathrm{ppm}$ after 45 hours. By increasing the temperature from $80^{\circ} \mathrm{C}$ to $100^{\circ} \mathrm{C}$, the maximum is reached after only 5 hours and the induction time (time before peroxides are detected) is much shorter.

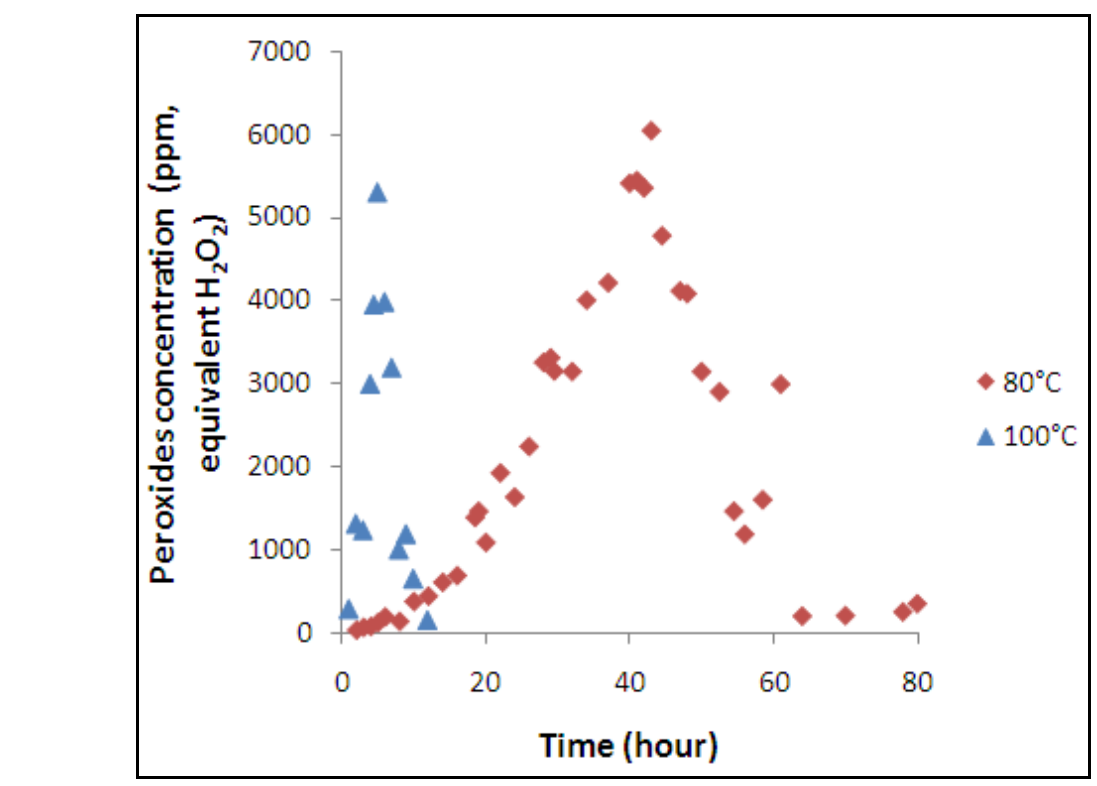

Figure 6.Peroxides concentration evolution at two operating temperatures

Figure 7 gives the pressure evolution for the experiment carried out at $80^{\circ} \mathrm{C}$ : pressure decreases in the first period, when peroxides are formed, and increases when the peroxide concentration decreases. According to Naito et al. [11], it is assumed that the pressure decrease corresponds to the oxygen consumption by the ether, the beginning of the autoxidation chain reaction, and the pressure increase corresponds to the formation of volatile products from peroxide decomposition. It has been indeed verified that the pressure drop is approximately equal to the initial concentration of oxygen in the autoclave. 


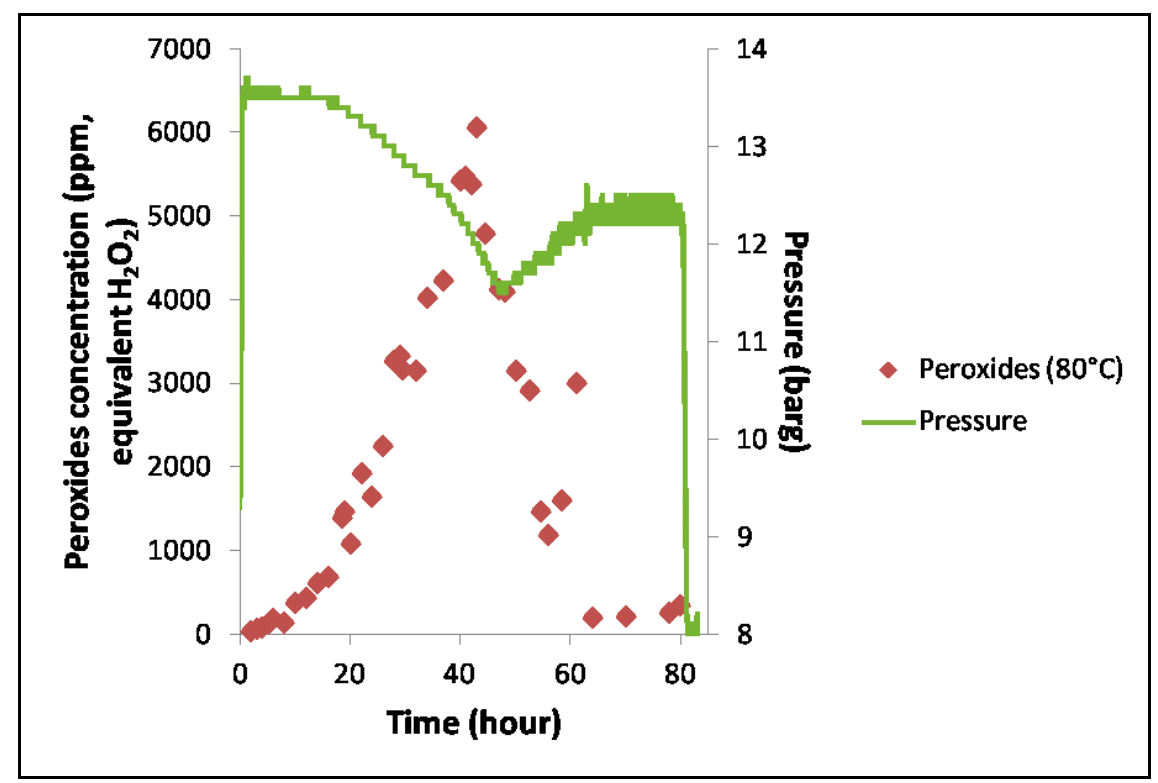

Figure 7.Peroxides concentration and pressure evolution at $80^{\circ} \mathrm{C}$

In this configuration and at this temperature, less than $1 \mathrm{~mL}$ of DEE should be vaporized in the autoclave. Nevertheless, the DEE is in excess relative to oxygen in gas phase. Thus the reaction could then occur either in the gas phase or liquid phase.

Further experiments need to be performed to determine more precisely the kinetics and the mechanism:

- Other temperatures should be tested (for instance $60^{\circ} \mathrm{C}$ and $110^{\circ} \mathrm{C}$ ) in order to extrapolate the kinetics to lower temperature.

- Other products identified in the molecular modeling study should be quantified (ethyl acetate, ethanol, ethyl formate, methanol, acetaldehyde, methanol...).

- The influence of the initial volume of DEE could give some indications about the phase where the reaction occurs.

- Various initial oxygen concentrations should be tested to confirm the correlation between the oxygen consumption and the peroxide production.

Nonetheless, the overall objective of this work is to couple the experimental results with the results produced by molecular and kinetic modeling to obtain a kinetics model of the oxidation of DEE in storage conditions, allowing the detection of its instability at ambient temperature.

\subsection{Kinetics model vs. experiments}

With this aim, data generated by CHEMKIN simulations in the kinetics model developed and validated for the gas phase oxidation of diethyl ether were compared with the experimental peroxide concentration curves described in the previous paragraph. In particular, all simulation parameters (initial reactor pressure, reactor volume, reactant concentrations) were chosen to correspond to actual experimental conditions, and the concentration of hydroperoxides was recorded. The simulation was made using a temperature of $107^{\circ} \mathrm{C}$ and compared with the peroxide concentrations measured during the experiment at $100^{\circ} \mathrm{C}$. This comparison is reported in figure 8. 


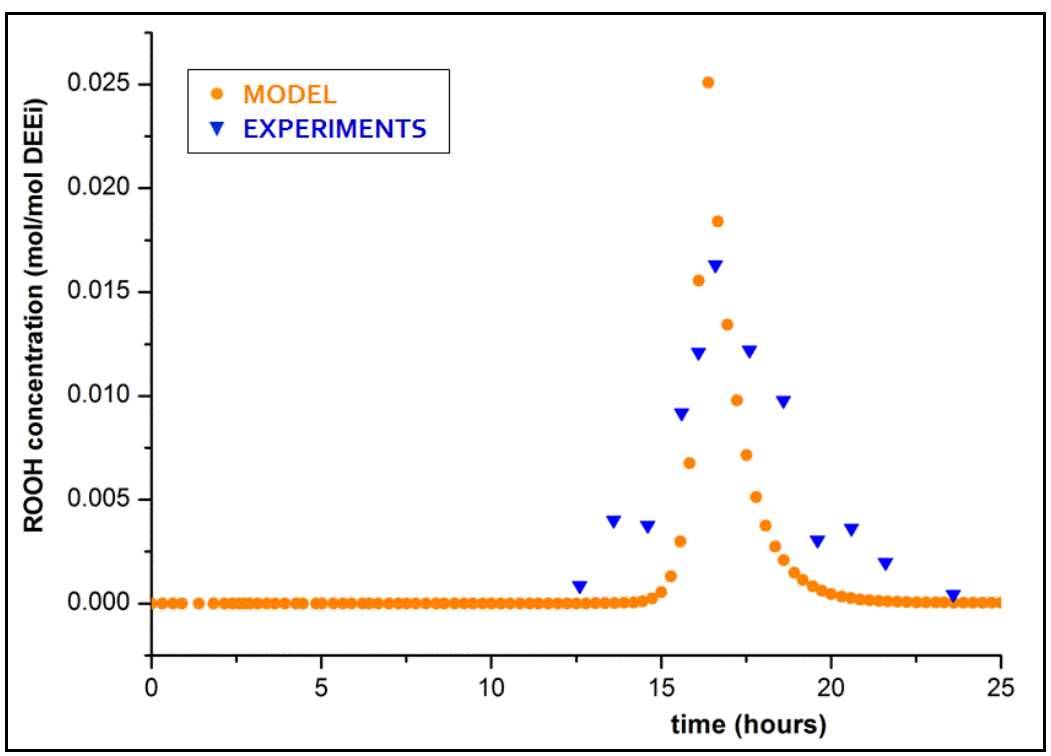

Figure 8. Comparison between peroxides concentration obtained from kinetics simulation at $107^{\circ} \mathrm{C}$ and experiments (at $100^{\circ} \mathrm{C}$ ). Experimental curve has been displaced by about 12 hours to facilitate the comparison

The results from the simulation $\left(\right.$ at $107^{\circ} \mathrm{C}$ ) predict a peak $\mathrm{ROOH}$ concentration coming 12 hours after the actual time of the peak observed in the experiment (at $100^{\circ} \mathrm{C}$ ). In figure 7 , the experimental results have been shifted in time to facilitate a comparison of the $\mathrm{ROOH}$ concentration. However, as seen before for the validation of the gas phase model, the ROOH concentrations predicted by the simulation and observed experimentally are quite similar.

These preliminary results encourage further improvement of the kinetics model using new experimental data obtained either under accelerated conditions or at ambient temperature with the goal to better reproduce the true behavior of DEE at storage conditions.

\section{Conclusions}

Two different but complementary theoretical approaches have been used to study the oxidation process of diethyl ether: an in-depth theoretical study based on quantum chemistry calculations and a kinetics study. Concerning the reaction mechanism, both approaches show that in presence of molecular oxygen, reaction pathways involving $\mathrm{O}_{2}$ are favored with respect to unimolecular decomposition. The kinetics model has been obtained and validated for the oxidation of DEE in the gas phase from experimental data obtained from the literature: the model reproduces well the selectivity of product blend but is less efficient for reaction time. This problem in predicting the time of reaction was also observed when comparing peroxide concentrations obtained at approximately $100^{\circ} \mathrm{C}$ from simulation and from preliminary oxidation tests performed with autoclaves at INERIS. However, the kinetics model is able to predict suitably the concentration of peroxides produced during the oxidation process of DEE. We can expect significant improvement in the kinetics model by taking into account the results of ongoing tests to quantify other molecular species. The objective of experimental study is also to extrapolate measurements of DEE oxidation products towards conditions of normal storage. From a risk point of view, this model is expected to determine accurately both peroxide concentrations and time of maximal 
concentration in order to predict potential instability and better manage industrial and laboratory risks of this chemical.

\section{References}

[1] R. Robertson, Chem. Ind., 1933, 52, 274.

[2] G. T. Morgan, R. H. Pickard, Chem. Ind., 1936, 55, 421.

[3] A. G. Davies, J. Roy. Inst. Chem., 1956, 80,386-389.

[4] V. J. Karnojitzky, Chim. Ind., 1962, 88, 233-238.

[5] S. L. Fischer, F. L. Dryer, H. Curran, Int. J. Chem. Kinet., 2000, 32, 713-740.

[6] J. Sehested, T. Mogelberg, T. J. Wallington, E. W. Kaiser, O. J. Nielsen, J. Phys. Chem., 1996, 100, 17218-17225.

[7] A. Andersen, E. A. Carter, J. Phys. Chem. A, 2003, 107, 9463-9478.

[8] K. C. Saloojaa, Combust. Flame, 1965, 9, 33-41.

[9] A. Lemay, C.Ouellet, Can. J. Chem., 1955, 33, 1316-1327.

[10] D. J. Waddington, Proc. R. Soc. London, Ser. A, 1959,252, 260-272.

[11] M. Naito, C. Radcliffe, Y. Wada, T. Hoshino, L. Xiongmin, M. Arai, M. Tamura, J. Loss Prev. Process Indust., 2005, 18, 469-473.

[12] J. J. Orlando, Phys. Chem. Chem. Phys. , 2007, 9, 4189-4199.

[13] A. C. Hamstead,L. S. VanDelinder, J. Chem. Eng. Data, 1960, 5, 383-386.

[14] T. C. Zhang, J. Wang, T. Yuan, X. Hong, L. D. Zhang, F. Qi, J. Phys. Chem. A, 2008, 112, 10487-10494.

[15] Semenov, N., Chemical Kinetics and Chain Reactions. Oxford University Press ed.; London, 1935.

[16] S. Di Tommaso, P. Rotureau, O. Crescenzi, C. Adamo, Phys. Chem. Chem. Phys., 2011, 13, 14636-14645.

[17] A. D. Becke, J. Chem. Phys., 1993, 98, 1372-1377.

[18] M. J. Frisch et al., Gaussian 03, 2004.

[19] C. Gonzalez, J. J. W. McDouall and H. B. Schlegel, J. Phys. Chem., 1990, 94, 7467-7471.

[20] K.J. Laidler, M.C. King, J. Phys. Chem., 1983, 87, 2657-2664.

[21] E. Wigner, Z. Phys. Chem., 1932, 19, 203-216.

[22] R.T. Skodje, D.G. Truhlar, J. Phys. Chem., 1981, 85, 624-628.

[23] C. Eckart, Phys. Rev., 1930, 35, 1303-1309.

[24] E.T. Denisov and I.B. Afanas'ev, Oxidation and Antioxidants in Organic Chemistry and Biology, Taylor \& Francis, Boca Ratom, FL, 2005.

[25] V. Warth, F. Battin-Leclerc, R. Fournet, P.-A.Glaude, G.M. Côme, G. Scacchi, Comput. Chem. 2000, 24, 541 - 560.

[26] C. Muller, V. Michel, G. Scacchi, G.M. Come, J. Chim. Phys., 1995, 92, 1154-1178.

[27] R.J. Kee, F.M. Rupley, J.A. Miller, CHEMKIN II. A Fortran Chemical Kinetics Package for the Analysis of a Gas-phase Chemical Kinetics, Sandia Laboratories Report, SAND 89-8009B, 1993. 
Table I. Activation barriers in terms of enthalpies and Gibbs free energies (respectively $\Delta \mathrm{H}^{\ddagger}$ and $\Delta \mathrm{G}^{\neq}$in $\left.\mathrm{kcal} / \mathrm{mol}\right)$, products stabilization $(\Delta \mathrm{H}, \mathrm{kcal} / \mathrm{mol})$ and rate constants $\left(\mathrm{k}, \mathrm{s}^{-1}\right)$ obtained for the three main reactions of the DEE oxidation mechanism.

\begin{tabular}{|c|c|c|c|c|}
\hline REACTION & $\boldsymbol{\Delta} \mathbf{H}^{\boldsymbol{*}}$ & $\boldsymbol{\Delta} \mathbf{H}$ & $\boldsymbol{\Delta} \mathbf{G}$ & $\boldsymbol{k}$ \\
\hline R. decomposition & 19.2 & 4.0 & 17.7 & 1.29 \\
ROO· isomerization & 17.2 & 14.8 & 19.3 & 0.88 \\
ROOH production & 13.3 & 12.8 & 23.7 & $2.3 \cdot 10^{-5}$ \\
\hline
\end{tabular}

